\title{
Zonificación edafoclimática para el cultivo de la papaya en Valencia Córdoba - Colombia
}

\author{
Edaphoclimatic zoning for growing papaya in \\ Valencia Cordoba - Colombia
}

\author{
Ricardo Leon Duran ${ }^{1}$, Enrique Miguel Combatt Caballero ${ }^{2 *}$, José Vicente Villalba Arteaga ${ }^{3}$ \\ Janer Miguel Polo Santos ${ }^{4}$, Robert Smith Valencia Agressoth ${ }^{5}$
}

Recibido para publicación: Septiembre 16 de 2017 - Aceptado para publicación: Mayo 24 de 2018

\begin{abstract}
RESUMEN
En este estudio se evaluaron y clasificaron los recursos climáticos y edáficos de la zona Norte del municipio de Valencia Córdoba, usando geoestadística y sistemas de información geográfica (SIG). En el componente climático se usaron datos de precipitación, temperatura, humedad relativa y radiación solar de cinco estaciones meteorológicas. Para el componente edafológico se realizaron 119 análisis de suelos de dos asociaciones productoras de papaya y plátano de la zona. La cartografía de distribución espacial de las variables se realizo por método de interpolación de la distancia inversa ponderada con el programa ArcMap; se elaboraron mapas de variables espacializadas en una escala 1:250.000. Mediante este procedimiento fueron calculadas áreas delimitadas por isolíneas en las variables de clima y suelo. En la cartografía de áreas con aptitud productiva se usó una escala de cuatro categorías con las variables $\mathrm{pH}$, M.O., textura, $\mathrm{P}$ y Al, las cuales fueron A1: Muy óptima, A2: óptima, A3: ligeramente óptima y A4: moderadamente óptima. Como resultado se obtuvieron: zonas muy óptimas (A1), 4,6\% equivalentes a aproximadamente a 3.050 ha, ubicadas en la parte norte del área de estudio, zonas óptimas (A2) el 55\%, con 36.440,7 ha, ubicadas en el centro del área de estudio, las zonas ligeramente óptimas (A3) el 35,5\%, con 23.533,6 ha, ubicadas en la parte suroriental y Zonas moderadamente óptimas (A4) el $4,7 \%$, con $3.156,5$ ha, ubicadas en el sector suroccidental de la zona estudiada. Esta clasificación es fundamental en la proyección agropecuaria de la zona y en en los proyectos de ordenamiento territorial.
\end{abstract}

Palabras clave: Zonificación, edafología, calentamiento global, Carica papaya, sistemas de información geográfica.

\begin{abstract}
This study involves the evaluation and classification of climate and soil resources in Valencia Cordoba, using geographic information systems. Climate component data from five meteorological stations with records of precipitation, temperature, relative humidity and solar radiation were used. Edafológic component data were obtained from 119 soils analyzes of the growing of papaya. To generate mapping spatial distribution of climatic and soil variables interpolation method of inverse distance weighted incorporated into the ArcMap program was used, this process led to the mapping of variables spatialized on a scale of 1: 250,000. By this method delimited by isolines in climate and soil variables they were calculated areas. To perform the mapping of areas with productive capacity a scale of four categories that involve the variables of soil $\mathrm{pH}, \mathrm{MO}$, texture, $\mathrm{P}$ y Al, which privileges the A1 was used: Very best, A2: excellent, A3: A4 optimal and slightly: moderately optimal. As a result we obtained the very best areas (A1), representing $4.6 \%$ $(3,050 \mathrm{ha})$, located in the northern part of the area that optimal areas (A2) comprise 55\% (36,440.7 ha), located in the central sector of the reference area, slightly optimal areas (A3) $(23,533.6 \mathrm{ha})$ and represent $35.5 \%$ of the total area, located in the southeast part and $4,7 \%$, (3156.5 ha) has been categorized as slightly optimal (A4) are located in the southwestern part of the study area.
\end{abstract}

Key words: Zoning, edaphology global warming, Carica papaya, geographic information systems.

\footnotetext{
${ }^{1}$ M. Sc. Ciencias Ambientales. Córdoba, Colombia. Correo: ricardoleonduran@hotmail.com

2 Ph.D. Docente Universidad de Córdoba. Carrera 6ª . No. 76-103 Montería. Correo: ecombatt@correo.unicordoba.edu.co Córdoba, Colombia. Autor para correspondencia

3* M. Sc. Docente Universidad de Córdoba. Carrera 6ª . No. 76-103 Montería. Correo: jvvillalva@correo.unicordoba.edu.co Córdoba, Colombia.

${ }^{4}$ M. Sc. en Biotecnología. Correo jpolo@correo.unicordoba.edu.co Córdoba, Colombia.

${ }^{5}$ Ingeniero Agrónomo. Candidato a M. Sc en Ciencias Agronómicas. Valencia@fca.edu.co
} 


\section{INTRODUCCIÓN}

Carica papaya es un fruto con propiedades antioxidante, vitaminas y fibra. A nivel mundial. Colombia, es el sexto pais productor a nivel mundial (FAO, 2010). De acuerdo al Departamento administrativo Nacional de Estadística(DANE,2016), ensuEncuestaNacional Agropecuaria, durante el 2015, la producción a nivel nacional fue de 105.459 toneladas de papaya, con rendimientos promedios de 29,7 t ha-1; siendo el departamento de Córdoba el principal productor con 31.563 toneladas, seguido por el Valle del Cauca, Meta y Nariño.

La comercialización de papaya desde Colombia al exterior presenta importantes logros al obtener en el 2010, la licencia para la exportación de papaya a Estados Unidos y la declaración de la Zona Norte del Valle del Cauca, Tierralta y Valencia en el Alto Sinú del departamento de Córdoba como área de baja prevalencia de Mosca de la Fruta (ICA, 2011; Gómez, 2013).

Existe una potencialidad en el cultivo, dado a las condiciones geográficas que presenta el área de estudio, en los trópicos hay especies frutales de valor que tienen una distribución geográfica restringida y que potencialmente podrían convertirse en cultivos importantes. Entre las especies más prometedoras están: guanábana (Annona muricata), caramboloa (Averrhoa carambola), pejibaye (Bactris gasipaes), papaya (Carica papaya), entre otras (León, 2013).

El cultivo de papaya requiere suelos con textura franca o franco-arenosa, bien aireados, permeables, profundos (Campostrini y Glenn, 2007). Sin embargo, el cultivo se adapta bien a diferentes tipos de suelo, siempre que tengan buen drenaje. Por lo que suelos pesados, compactados o mal aireados, no son recomendablesparaelcultivo(Huesoetal.,2015).

El $\mathrm{pH}$ del suelo para el cultivo debe estar entre 5 y 7 , siendo el rango óptimo de 5,5 a 6,5. En suelos ácidos $(\mathrm{pH}=5$ - 5,5) es conveniente realizar encalado para mejorar el desarrollo de la planta y los rendimientos (Hueso et al., 2015). En Colombia el cultivo de papaya se encuentra distribuido en suelos con $\mathrm{pH}$ de 5 a 7,5 (Arango et al., 1999).

La temperatura es el factor más limitante para el desarrollo de una especie. Según Campostrini y Gleen (2007), cambios en las condiciones de temperatura, luminosidad y radiación pueden acarrear pérdidas en el período de formación. El rango de temperaturas óptimo para el desarrollo de la papaya se encuentra entre los 21 y $33{ }^{\circ} \mathrm{C}$, siendo $25{ }^{\circ} \mathrm{C}$ la temperatura ideal para el cultivo. Temperaturas por debajo de los $20{ }^{\circ} \mathrm{C}$ o por encima de los $35{ }^{\circ} \mathrm{C}$ provocan serias alteraciones florales, que afectan a la producción y calidad de los frutos. Según Arango et al. (1999), el cultivo en Colombia se desarrolla desde el nivel del mar hasta los 1.600 metros de altitud, con temperaturas entre 17 y 32 oC y para su desarrollo se encuentra en regiones con temperaturas promedios de 22 a $28{ }^{\circ} \mathrm{C}$.

Periodos luminosos del día aproximadamente de 12 horas, donde la energía radiante puede alcanzar la densidad de flujo de $2.000 \mu \mathrm{mol}$ m-2 s-1, puede cambiar la conductividad estomática del cultivo y disminuir la tasa de asimilación del carbono (Jeyakumar etal., 2007).

La pluviosidad promedio para el cultivo deben estar entre 1.500 y 2.000 mm anuales, bien distribuidos, con una humedad relativa del entre el 60 y el $85 \%$. Vientos fuertes pueden causar daños en las hojas, reduciendo el área de fotosíntesis y exponen los frutos al sol, provocando también su caída (Clemente y Marler, 2001). La falta de humedad dificulta el cuajado de frutos y provoca la caída prematura de las hojas (Hueso et al., 2015).

En el ámbito de la planificación agrícola, la zonificación agroecológica es una de las 
principales herramientas empleadas para disminuir los riesgos climáticos a los que está sometida la agricultura, la misma tiene como objetivo otorgar espacios a cultivos atendiendo a sus exigencias edafoclimáticas para garantizar en gran medida el crecimiento, desarrollo y rendimiento de los núcleos agroproductivos (Venero, 2014).

Una zonificación edáfica y agroclimática corresponde a la detección de áreas geográficas homogéneas en sus características climáticas y edáficas para la producción de cultivos. La zonificación agroecológica (ZAE) se refiere a la división de la superficie de la tierra en unidades más pequeñas, que tienen características similares relacionadas con su aptitud, con la producción potencial y con el impacto ambiental (FAO, 1997). Los estudios de zonificación agroecológica de cultivos, son muy importantes en la delimitación de áreas, ya que con ellos es posible definir qué cultivos tienen mayor potencial de producción. Las investigaciones más avanzadas han incorporado bases de datos enlazadas con sistemas de información geográfica (SIG), relacionadas con modelos estadísticos, que contienen múltiples aplicaciones potenciales en el manejo de los recursos naturales y la planificación del uso de la tierra (Jiménez et al., 2004).

Otros estudios han utilizado como criterio la combinación de tres parámetros: balance hídrico, producción de biomasa y calidad de suelos (Espinosa y Roquera, 2007); también han desarrollado metodologías donde aplican criterios de decisión ofrecidos por especialistas en ciencias agrícolas (Garea et al., 2008; Suárez et al., 2013).

Los datos geográficos son la base en cualquier SIG; la dificultad en la recolección de algunos datos y lo perentorio de su actualidad provoca que sea este elemento el más costoso de todos los componentes de un sistema de información geográfica, ya que estos deben responder a tres componentes principales: espacial, temática y temporal. Un SIG, es aquel capaz de dar respuesta al objetivo para el cual es diseñado, debe conjugar cuatro componentes: una serie de dispositivos (máquinas electrónicas), programas (SIG), conjunto de datos geográficos (la base de datos espaciales) y expertos en el manejo de los tres elementos previos (Moreno, 2005). Este conjunto de información Amplían enormemente las posibilidades de análisis que brindan los mapas convencionales, además de facilitar su almacenamiento y visualización (Chuvieco, 2002).

El objetivo del estudio consistió en construir un mapa de aptitud agroecológica que identifique las zonas con alto potencial productivo para el cultivo de papaya en el municipio de Valencia Córdoba, a través de elaboración de mapas de distribución espacial de variables climáticas y edafológicas, determinando el área óptima para la siembra del cultivo, dada sus características fisiológicas y requerimientos nutricionales, para su expoltación buscando la sostenibilidad y competitiividad en el sector productivo.

\section{MATERIALES Y MÉTODOS}

El área de estudio posee una extensión de 66181,5 ha correspondiente al 72,2\% del área total del municipio de Valencia y ubicada en su parte norte como se observa en la figura 1. El Municipio de Valencia se encuentra localizado en la parte sur occidental del departamento de Córdoba, entre el Valle del Sinú y la Serranía de Abibe, a $76^{\circ} 09^{\prime}$ Longitud Oeste y $8^{\circ} 16^{\prime}$ de Latitud Norte. Con una extensión de 916,4 km² que representan el 3\% del área departamental. Limita al norte con el Municipio de Montería, al sur con el Municipio de Tierralta, al este con los Municipios de Montería y Tierralta y al oeste con el Municipio de San Pedro de Urabá.

En el análisis de la variable de clima se utilizaron datos proporcionados por el Instituto de Hidrología, Meteorología y Estudios 
Ambientales (IDEAM) de cinco estaciones distribuidas en la región y cerca al área de estudio. Los datos obtenidos de ellas son promedios mensuales multianuales que corresponden a los parámetros de precipitación, temperatura, humedad relativa y brillo solar.
Los datos faltantes en cada una de las series de cada variable climática fueron completados realizando previamente una matriz de análisis de correlación entre la estación faltante y las estaciones completas en el mismo período de tiempo de registros.

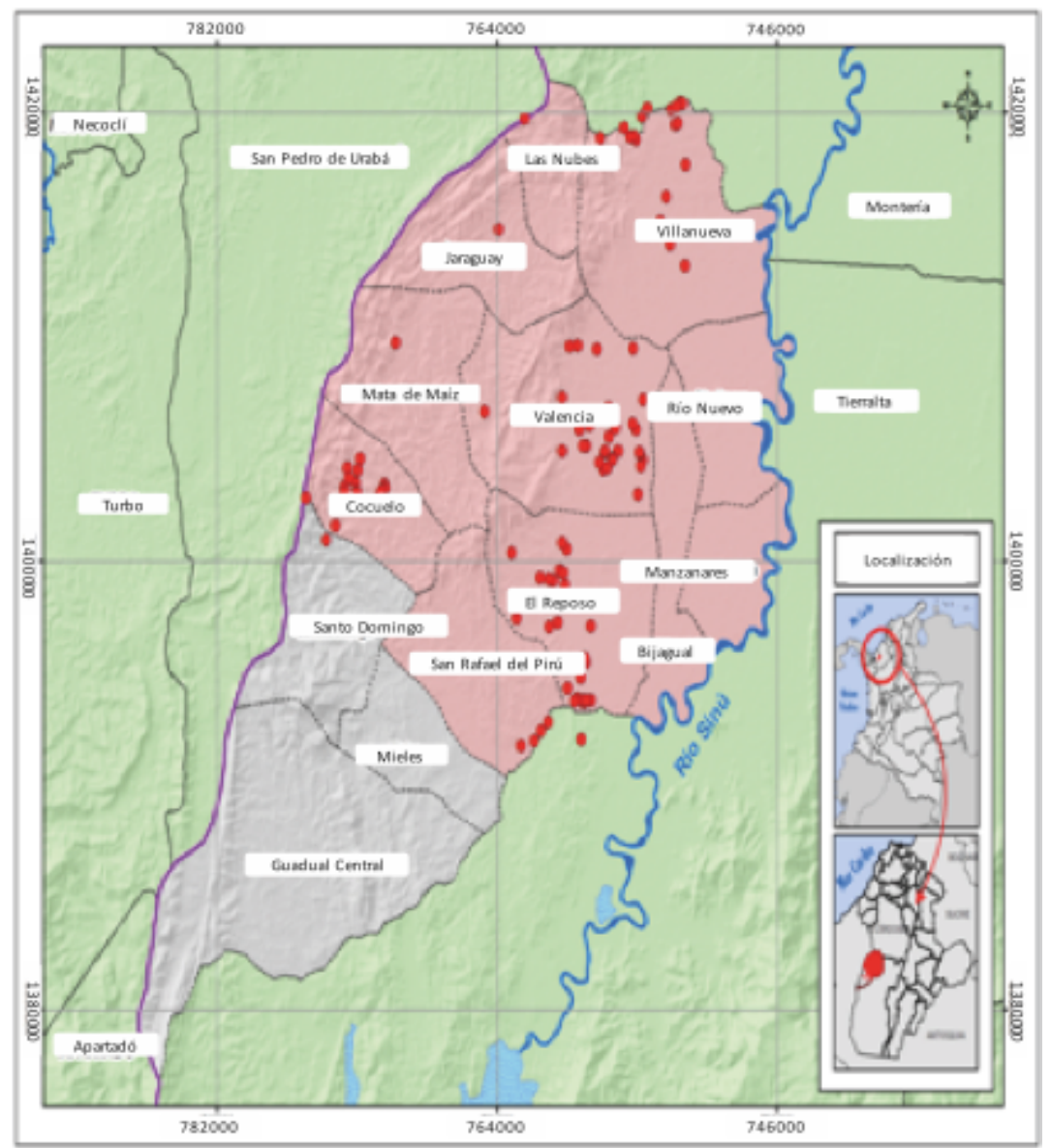

\begin{tabular}{|c|c|c|c|}
\hline \multicolumn{4}{|c|}{ Área de Estudio y Puntos de Muestreo } \\
\hline \multicolumn{4}{|c|}{$\begin{array}{c}\text { Escala } \\
1: 250.000\end{array}$} \\
\hline \multicolumn{4}{|c|}{8} \\
\hline \multicolumn{4}{|c|}{ Convenciones } \\
\hline \multicolumn{4}{|c|}{$\begin{array}{l}\text { Límite Corregimental } \\
\text { Límite Municipal } \\
\text { Límite Departamental } \\
\text { Cuenajes Dobles } \\
\text { Cuesos de Agua }\end{array}$} \\
\hline & $\begin{array}{l}\text { Le } \\
\text { Puntos de Muestr } \\
\text { Área de Estudio }\end{array}$ & enda & \\
\hline No. & Corregimientos & Área (ha) & Porcentaje (\%) \\
\hline 1 & San Rafael del pirú & $6.514,77$ & 9,84 \\
\hline 2 & El reposo & $7.851,84$ & 11,86 \\
\hline 3 & Bijagual & $4.201,26$ & 6,35 \\
\hline 4 & Cocuelo & $4.148,98$ & 6,27 \\
\hline 5 & Mata Maíz & $7.152,78$ & 10,81 \\
\hline 6 & Valencia & $9.975,03$ & 15.07 \\
\hline 7 & Las Nubes & $2.691,40$ & 4,07 \\
\hline 8 & Jaraguay & $5.871,95$ & 8,87 \\
\hline 9 & Villanueva & $9.796,54$ & 14,80 \\
\hline 10 & Manzanares & $3.502,40$ & 5,29 \\
\hline 11 & Río Nuevo & $4,474,56$ & 6,76 \\
\hline & TOTAL & $66.181,5$ & 100 \\
\hline \multicolumn{2}{|c|}{ Información Cartográfica } & \multicolumn{2}{|c|}{ Información Temática } \\
\hline \multirow{3}{*}{\multicolumn{2}{|c|}{$\begin{array}{l}\text { Proyección: Conforme de } \\
\text { Gauss } \\
\text { Origen: Bogotá } \\
\text { Dalum: Magna Sirga } \\
\text { Colombia } \\
\text { Falso Norte: } 1000000 \mathrm{mN} \\
\text { Falso Este: } \\
1000000 \mathrm{mN}\end{array}$}} & \multicolumn{2}{|c|}{$\begin{array}{l}\text { Recolección de Información } \\
\text { Primaria en Campo }\end{array}$} \\
\hline & & \multicolumn{2}{|c|}{ Diseño: Ricardo león Durán } \\
\hline & & \multicolumn{2}{|c|}{ Fecha: Octubre de 2015} \\
\hline
\end{tabular}

Figura 1. Localización del área de estudio y puntos de muestreo.

Estos análisis de correlación fueron realizados con ayuda del programa Excel; con la realización de los diferentes análisis de correlación se determinó con qué estación se completaron los datos faltantes en cada variable climática. Con la estación carente de datos y la que mostró el mayor coeficiente de correlación en cada variable se llevó a cabo un análisis de regresión lineal simple para determinar el modelo o ecuación lineal que liga las dos series, mediante el formato que muestra la siguiente ecuación:

$$
Y=\varphi \quad X \pm C
$$

donde:

Y: representa la serie de datos faltante de la variable climatológica

$\mathbf{X}$ : serie de datos de la estación de referencia $\boldsymbol{\varphi}$ : coeficiente de regresión

C: es la constante

La zonificación edafológica se colectaron un total de 119 análisis de suelos, 53 suministrados por la agremiación de productores de papaya (APPALSI) y 66 por la Asociación de Desplazados Nuevo Futuro (ASODENUF), dedicada a la producción de plátano, ambas 
con sede y actividad productiva actual en el municipio de Valencia. De estos análisis se tomaron parámetros de $\mathrm{pH}$, materia orgánica, textura y contenidos de fósforo y aluminio.

La información se procesó por medio del paquete estadístico SAS (versión 9.1), realizando progresivamente Análisis Exploratorio de datos, posteriormente se realizó un análisis de varianza para observar si existían diferencia entre localidades y se culminó con una prueba de comparación de medias de Duncan, para determinar entre qué localidades se hallaban diferencias en relación con los variables $\mathrm{pH}$, materia orgánica, elementos mayores y elementos menores. Para el análisis de la información climática se tomaron como referencia los datos multianuales de la estaciones de referencia en las variables de temperatura, precipitación, humedad relativa y brillo solar para diferentes períodos de registros a nivel de estación y de variable climática.

Para la elaboración de los mapas de distribución espacial de las variables de clima y suelo, se realizaron procesos geoestadísticos utilizando software ArcMap 10.3 del paquete ArcGIS de la empresa ESRI. Los geoprocesos consisten en un sistema de mapeo computarizado que relaciona lugares con información climática y edafológica. La interpolación para el cálculo de isolíneas se realizó con el método de la Distancia Inversa Ponderada (IDW: inverse distance weighting), incluido dentro del programa ArcMap. La interpolación mediante distancia inversa ponderada determina los valores de celda a través de una combinación ponderada linealmente de un conjunto de puntos de muestra. La ponderación es una función de la distancia inversa. La superficie que se interpola debe ser la de una variable dependiente de la ubicación. Para la realización del mapa de isolíneas en donde se discrimina y se produce la zonificacíon por categoría de aptitud productiva, se utilizaron los rangos de las variables edáficas (Tabla 1). En esta tabla no se incluyeron variables climáticas, ya que la variación de estos parámetros dentro de la zona objeto de estudio es muy pequeño y los rangos de fluctuación están dentro de las necesidades del cultivo de papaya, por tanto se obviaron para la elaboración de las categorías. Las categorías son: A1: Muy óptima, A2: óptima, A3: ligeramente óptima y A4: moderadamente óptima.

\section{RESULTADOS Y DISCUSIÓN}

Componente climático. La distribución de la precipitación anual incidente en el área de estudio, muestra que aumenta del Norte, siendo las localidades de Las Nubes y Vilanueva donde la pluviosidad es más baja.

Las altas precipitaciones observadas en el sur, se pueden presentar por influencia de la Cordillera Occidental (Serranía de Abibe), ya que la zona de estudio, se encuentra en el pie de monte del Parque Nacional Natural Paramillo. Los resultados de este estudio en cuanto a la precipitación son acordes con lo emitido por Palencia et al. (2006), quienes afirman que la precipitación en el municipio de Valencia varía de Norte a Sur con valores que oscilan entre 1400

Tabla 1. Categorías de aptitud productiva del suelo de acuerdo a rango de variables edáficas.

\begin{tabular}{cccccc}
\hline Categoría & $\mathbf{P H}$ & $\begin{array}{c}\text { MO } \\
\mathbf{1}\end{array}$ & $\begin{array}{c}\mathbf{P} \\
\mathbf{m g ~ k g - 1}\end{array}$ & $\begin{array}{c}\text { Al } \\
\text { Cmolc kg-1 }^{-1}\end{array}$ & Textura \\
\hline A1 & $>6$ & $>3,5$ & $>25$ & $<0,4$ & $\mathrm{ArA}-\mathrm{FAr}$ \\
A2 & $5,5-6,0$ & $3,0-3,5$ & $15-25$ & $0,4-0,7$ & $\mathrm{Ar}-\mathrm{FArA}$ \\
A3 & $5,0-5,5$ & $2,0-3,0$ & $10-15$ & $0,7-1,0$ & FA - FArL \\
A4 & $<5$ & $<2,0$ & $<10$ & $>1,0$ & $\mathrm{~A}-\mathrm{L}$ \\
\hline
\end{tabular}

*ArA: arcillo arenoso; FAr: francoarcilloso; Ar: arcilloso; FArA: franco arcillo arenoso; FA: franco arenoso; FArL: franco arcillo limoso; A: arenoso; L: limoso. 
- 2400 mm por año, siendo el sur del municipio donde se presentan las mayores precipitaciones.

La distribución de la temperatura, muestra una variación de Sur a Norte contraria a la precipitación. Para la elaboración del mapa de isotermas se consideraron variaciones de $0,05{ }^{\circ} \mathrm{C}$ ya que la temperatura es un parámetro que presenta muy poca variación incluso a escala departamental. Esta forma de variación de la temperatura sugiere estrategias distintas para los agricultores del sector norte, en donde la precipitación no es escasa pero cuantitativamente es la menor, además la temperatura es la más alta de la zona, con estas condiciones es posible que se puedan presentar problemas de déficit hídrico, lo que indica que es necesario crear e implementar sistemas de riego para eventuales temporadas de altas temperaturas y pocas precipitaciones. La distribución espacial de la humedad relativa indica que la variación ocurre desde la parte Nororiental hacia el Sur y Suroccidente de la zona de estudio.

El comportamiento espacial de la humedad en esta zona indica que está estrechamente relacionada con la temperatura y brillo solar, ya que los patrones de distribución son realmente muy similares. La humedad relativa es un parámetro climatológico relacionado a través de diversos mecanismos físicos con la nubosidad, precipitación y de forma muy especial con la temperatura; la cantidad de agua en forma de vapor que puede encontrarse en la atmósfera es función directa de la temperatura (Barros y troncoso, 2010). La humedad refleja los periodos de máxima y mínima precipitación, siendo baja en los meses de menos lluvias y alta en los meses de mayor Iluvia. Palencia et al. (2006), afirman que la humedad relativa en el departamento de Córdoba oscila entre los $80-86 \%$ y en el municipio de Valencia varía entre el $83-84 \%$ en el Norte del municipio cubriendo gran parte del mismo y llegando hasta $85 \%$ en el Sur. La tendencia espacial del brillo solar ocurre desde el sur en el corregimiento San Rafael del Pirú hacia el nororiente de la zona objeto de estudio. Las localidades que acumulan más horas de sol mensuales son Villanueva y Rio Nuevo. El brillo solar en el municipio de Valencia oscila entre 1800 - 2000 horas al año, siendo la parte norte del municipio la que está expuesta a menor insolación y la parte sur la que acumula más horas de brillo solar durante el año (Palencia et al., 2006).

Componente edafológico. El análisis descriptivo para el fósforo, arrojó que los contenidos de este elemento oscilan entre 1,2 y $28,4 \mathrm{mg} \mathrm{kg}^{-1}$ con un valor promedio de 10,56 $\mathrm{mg} \mathrm{kg}^{-1}$, y una desviación estándar de 6,3 (Tabla 2). De acuerdo a la prueba de comparación de medias de Duncan, se determinó que las localidades con mayor contenido de este elemento fueron Tinajones y Reposito que tienen contenidos superiores a $14,5 \mathrm{mg} \mathrm{kg}^{-1}$, sin diferencias estadísticamente significativas con las localidades que contienen en promedio hasta $8,9 \mathrm{mg} \mathrm{kg}^{-1}$. Las localidades con menor contenido de fósforo fueron Las Piedras y Bijagual con 4,54 y 5,32 $\mathrm{mg} \mathrm{kg}^{-1}$ respectivamente. Las localidades con contenidos medios de fósforo fueron Reposo y Nuevasión con valores que oscilaron entre 10 y $12 \mathrm{mg} \mathrm{kg}^{-1}$.

La superficie con contenidos mayores a 25 mg $\mathrm{kg}^{-1}$ representa un área de 7,7 ha, que corresponde aproximadamente al 0,01\% del área total de estudio. 50,5\% del área presentó contenido de $\mathrm{P}$ dentro del rango 10 - $15 \mathrm{mg}$ $\mathrm{kg}^{-1}$ con 33.399 ha, similar a lo encontrado por IGAC (2009) y Combatt et al. (2012) en cuanto al contenido de este elemento en los suelos de Valencia (Figura 2a). Un estudio de caracterización química de macroelementos, realizado por Combatt et al. (2012), encontró que en la zona productora de plátano del municipio de Valencia los suelos presentan contenidos promedio de $\mathrm{P}$ con 27,6 $\mathrm{mg} \mathrm{kg}^{-1}$. 
Tabla 1. Comparación multiple de Duncan para los contenidos de fósforo $\mathrm{P}$, aluminio $\mathrm{Al3+}$, materia orgánica M.O. y niveles de acidez $\mathrm{pH}$, en las localidades del estudio, Municipio de Valencia, Departamento de Córdoba, Colombia.

\begin{tabular}{|c|c|c|c|c|}
\hline Localidades & $\begin{array}{l}\text { PH } \\
1: 1\end{array}$ & $\begin{array}{c}\mathrm{MO} \\
\%\end{array}$ & $\begin{array}{c}P \\
\mathrm{mg} \mathrm{kg}^{-1}\end{array}$ & $\begin{array}{c}\mathrm{Al} \\
\mathrm{Cmolc} \mathbf{k g}^{-1}\end{array}$ \\
\hline L1 & 5,92 a & 2,84 abcd & $14,98 \mathrm{a}$ & $0,17 \mathrm{~cd}$ \\
\hline L2 & $5,24 \mathrm{~cd}$ & $2,17 \mathrm{~d}$ & $14,76 \mathrm{a}$ & $0,64 a b c$ \\
\hline L3 & 5,39 bcd & $2,83 \mathrm{abcd}$ & $10,05 \mathrm{abcd}$ & $0,38 \mathrm{bcd}$ \\
\hline L4 & $5,34 \mathrm{~cd}$ & 2,47 bcd & $13,91 \mathrm{ab}$ & $0,38 \mathrm{bcd}$ \\
\hline L5 & $5,08 \mathrm{~d}$ & $2,53 \mathrm{bcd}$ & $11,85 \mathrm{abcd}$ & $0,38 \mathrm{ab}$ \\
\hline L6 & $4,98 \mathrm{~d}$ & 3,05 abc & 8,91 abcd & 0,94 a \\
\hline L7 & $5,27 \mathrm{~cd}$ & $2,40 \mathrm{bcd}$ & $13,06 \mathrm{ab}$ & 0,58 abcd \\
\hline L8 & 5,39 bcd & $2,34 \mathrm{~cd}$ & $12,44 \mathrm{abcd}$ & 0,55 abcd \\
\hline L9 & 5,38 bcd & $3,15 \mathrm{ab}$ & $6,45 \mathrm{~cd}$ & 0,29 bcd \\
\hline L10 & $5,71 \mathrm{abc}$ & $2,59 \mathrm{bcd}$ & $4,54 \mathrm{~d}$ & $0,05 \mathrm{~d}$ \\
\hline L11 & $5,59 \mathrm{abcd}$ & 3,39 a & $6,92 \mathrm{bcd}$ & $0,10 \mathrm{~d}$ \\
\hline L12 & 5,88 a & 2,91 abcd & $6,22 \mathrm{~cd}$ & $0,18 \mathrm{~cd}$ \\
\hline L13 & $5,63 \mathrm{abcd}$ & 2,86 abcd & $5,32 \mathrm{~d}$ & $0,15 \mathrm{~cd}$ \\
\hline Media & 5,46 & 2,7 & 10,57 & 0.39 \\
\hline $\mathrm{s}$ & 0,5 & 0,69 & 6,3 & 0,49 \\
\hline
\end{tabular}

L1:Tinajones, L2:Reposito, L3:Reposo, L4:Venado Cantarrana, L5:Nuevasión, L6:Jericó, L7:Barrial, L8:Pirú Arriba, L9:Buenavista, L10:Las Piedras, L11:Villanueva, L12:Las Flores y L13:Bijagual. *Letras similares en orden vertical no presentan diferencias estadísticas significativas

Los contenidos de fósforo tienen una marcada tendencia de aumentar de norte a sur y de occidente a oriente dentro del área de estudio.

Acidez intercambiable. De acuerdo con el análisis exploratorio para este parámetro, se observa que el promedio del contenido de $\mathrm{H}^{+}+\mathrm{Al}^{3+}$ fue de $0,39 \mathrm{cmol}_{\mathrm{c}} \mathrm{kg}^{-1}$, presenta desviación estándar de 0,49 y un coeficiente de variación de 0,49 . Los valores de $\mathrm{H}^{+}+\mathrm{Al}^{3+}$ en las localidades oscilaron entre 0 y 2,6 $\mathrm{cmol}_{\mathrm{c}} \mathrm{kg}^{-1}$. De acuerdo a la prueba de rangos múltiples de Duncan este elemento presentó diferencias estadísticamente significativas entre las localidades, mostrando mayor contenido en Jericó con 0,94 $\mathrm{cmol}_{\mathrm{c}} \mathrm{kg}^{-1}$. La localidad con menor contenido de $\mathrm{H}++\mathrm{Al} 3+$ fue Las piedras con $0,053 \mathrm{cmol}_{\mathrm{c}} \mathrm{kg}^{-1}$. Las localidades con contenido medios de acidez intercambiables (Al) fueron Reposo, Venado y Cantarrana con $0,38 \mathrm{cmol}_{\mathrm{c}} \mathrm{kg}^{-1}$ (Tabla 2). La distribución espacial del contenido de $\mathrm{H}^{+}+\mathrm{Al}^{3+}$ en localidades del municipio de Valencia fue de 1,61\% del área total de estudio, presentando contenido inferiores a $0,1 \mathrm{cmol}_{\mathrm{c}} \mathrm{kg}$ ${ }^{1}$, lo que equivale s 1.066 ha. Contenidos entre
$0,1-0,5 \mathrm{cmol}_{\mathrm{c}} \mathrm{kg}^{-1}$ representan el $76,3 \%$ con 48.742 ha, contenidos superiores a 2 cmolc kg- 1 representan el $0,05 \%$ con 30,8 ha (Figura $2 b$ ). La toxicidad por $\mathrm{Al}^{3+}$ es un factor importante, ya que limita el crecimiento de las plantas (Cristancho et al., 2010), sobre todo en suelos fuertemente ácidos con $\mathrm{pH}$ por debajo de 5,0 y el principal efecto de la toxicidad de $\mathrm{Al}^{3+}$ es la restricción del desarrollo radical (Rivera et al., 2016). En este estudio, se encontró una relación entrezonas dondelos contenidos dealuminioson altos (>a 0,7 $\mathrm{cmol}_{\mathrm{c}} \mathrm{kg}^{-1}$ ) y el $\mathrm{pH}$ es menor de 4,5.

La adición de materia orgánica puede reducir la fitotoxicidad por aluminio mediante la formación de complejos entre los compuestos orgánicos con el elemento (Hue et al., 1986; Kerven et al., 1989; Casierra y Aguilar, 2007). El ácido fúlvico es uno de los compuestos que con mayor efectividad reduce la totoxicidad del aluminio, por su capacidad para formar complejos con monómeros y polímeros del elemento. La aplicación de mulch y abonos verdes presenta también una gran efectividad en la reducción de los efectos tóxicos del aluminio en suelos ácidos (Casierra y Aguilar, 2007). 
León et al. - Zonificación edafoclimática en cultivo de papaya

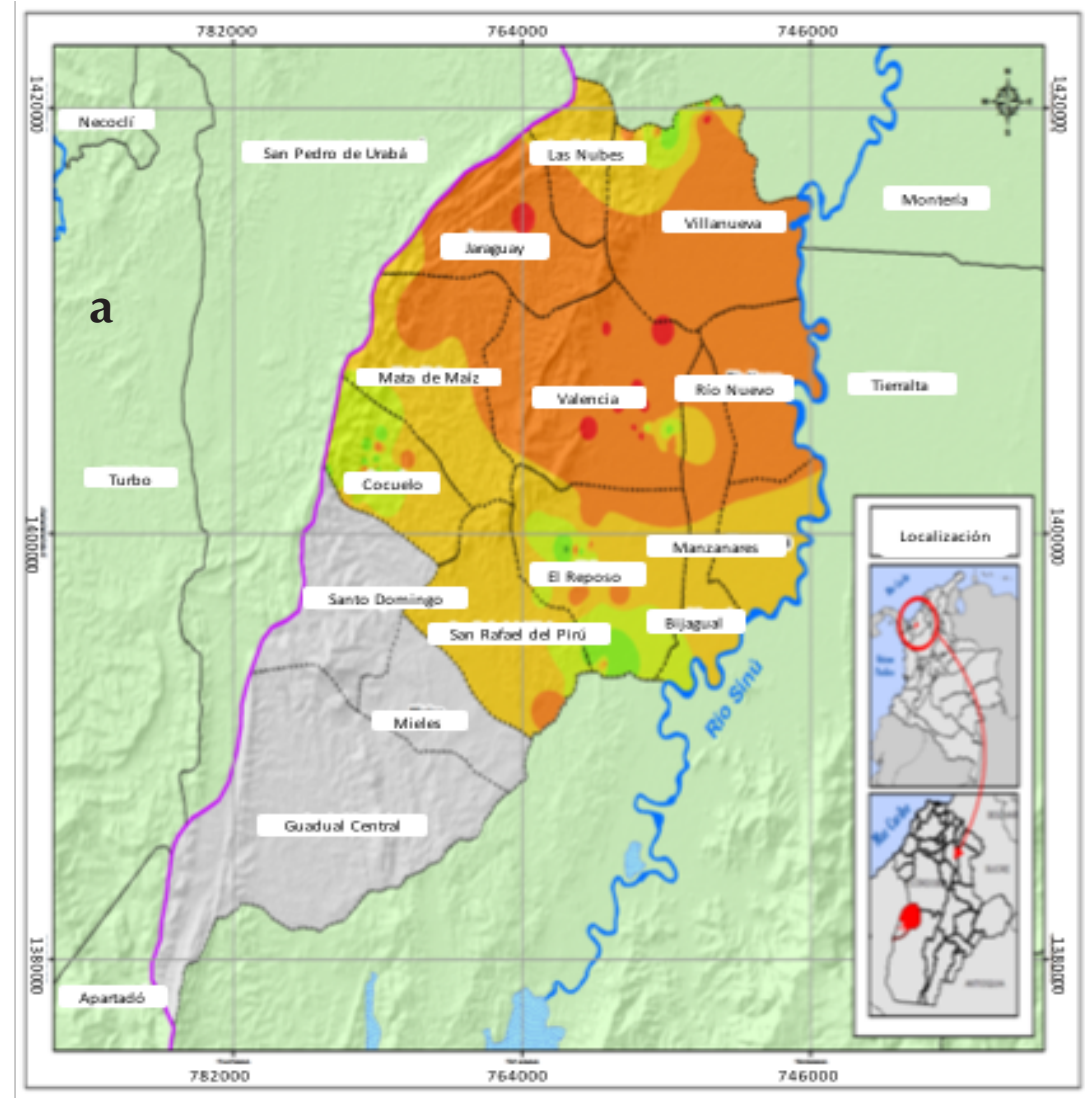

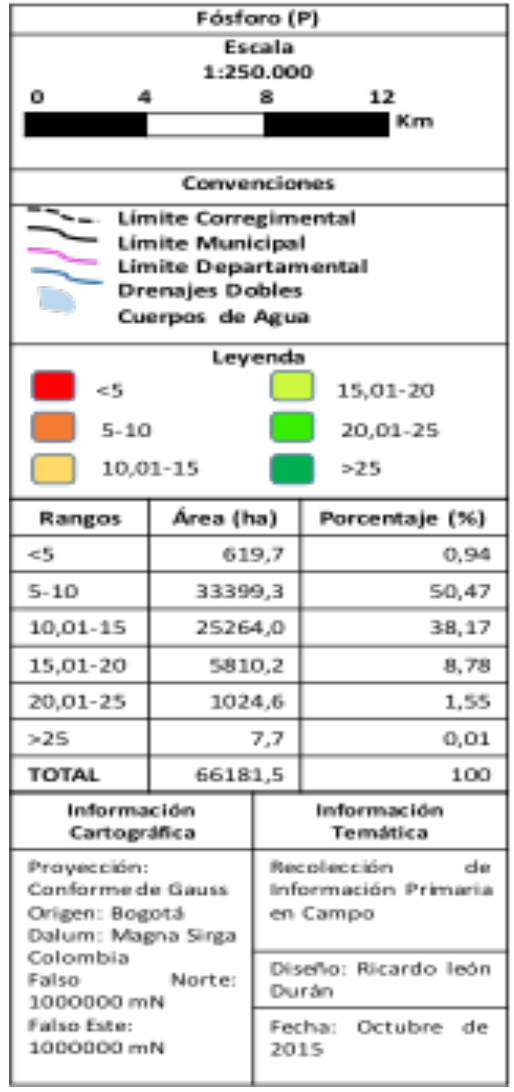

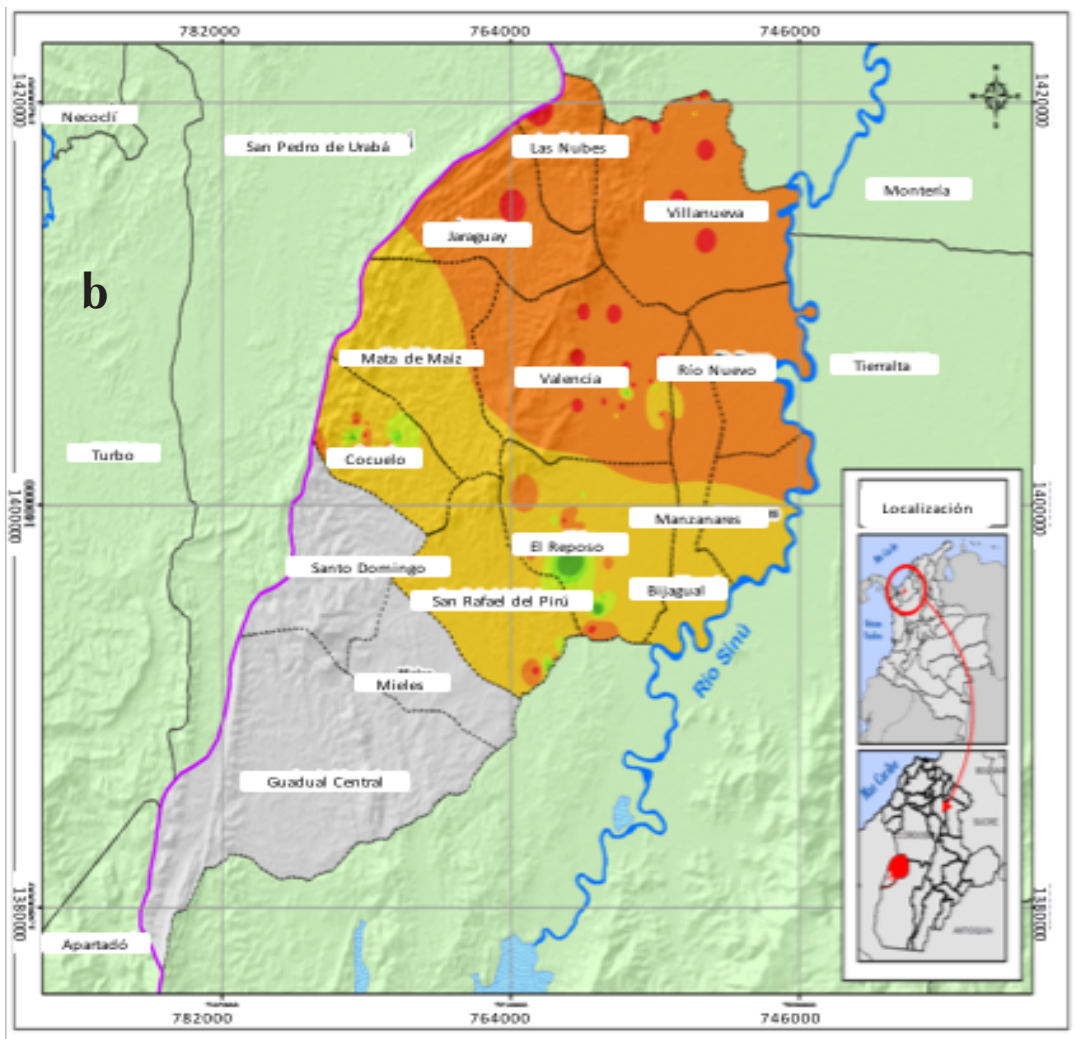

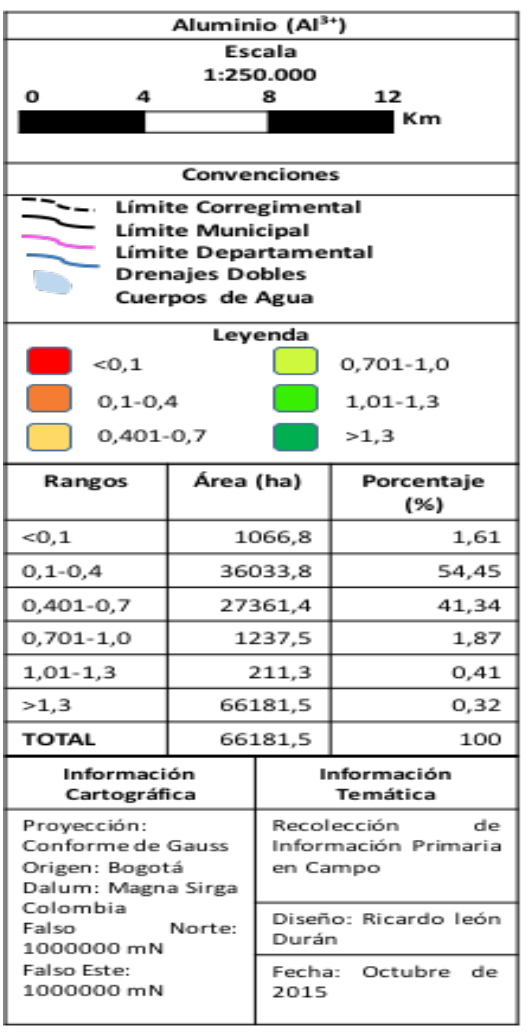

Figura 2. Contenidos (a y b) y distribución espacial de fósforo y aluminio en diferentes localidades del Municipio de Valencia, Córdoba, Colombia. 
pH. El análisis exploratorio de esta variable destaca que el valor promedio fue de 5,46, desviación estándar de 0,5 y un coeficiente de variación de 0,09. Esta distribución de datos osciló entre los valores 4,26 a un valor muy cercano a la neutralidad de 6,93 (Tabla 2). Este factor presenta gran variabilidad en sus niveles promedios entre las localidades, pero todas ella mostraron niveles por debajo de 6,0, lo que indica que en estas zonas predominan los suelos ligeramente ácidos a moderadamente ácidos. El pH más cercano a la neutralidad lo posee la localidad de Tinajones con 5,9 y el más bajo Jericó con una unidad por debajo de ésta, con 4,9. Las localidades con $\mathrm{pH}$ intermedio entre estos valores fueron las localidades de Reposo y Pirú Arriba (Figura 3a). La reacción del suelo o $\mathrm{pH}$, muestra que aproximadamente el 98\% del área total de estudio están ocupadas por zonas con pH entre 5,0 - 6,0 que con equivalente a 64.933 ha, indicando que los suelos de esta zona son prevalentemente ácidos. El pH presenta una distribución regular, aumentando de Sur a Norte. Es muy probable que este fenómeno ocurra porque el régimen de lluvias del municipio de Valencia es menor en el sector norte, lo que hace que la concentración de protones sea mayor repercutiendo en un $\mathrm{pH}$ de mayor valor, no obstante el promedio total de la zona de estudio indica tendencia a la acidez. Los factores que influyen en el valor del $\mathrm{pH}$ son diversos, entre los que podemos numerar el material parental, las precipitaciones por lixiviación de las bases calcio $\left(\mathrm{Ca}^{2+}\right)$, magnesio $\left(\mathrm{Mg}^{2+}\right)$, potasio $\left(\mathrm{K}^{+}\right)$y sodio $\left(\mathrm{Na}^{+}\right)$, disueltas en solución e intercambio de las bases adsorbidas al complejo de intercambio por $\mathrm{H}^{+}$(Vázquez, 2006).

Materia orgánica. En el análisis descriptivo para la variable de materia orgánica, se observa que ésta oscila con valores dentro del rango 1,02 a 4,2\%. Con una media de 2,7\%, la desviación estándar de 0,69 y un coeficiente de variación de 0,26 (Tabla 2). Combatt et al. (2012), para esta zona obtuvo una media para el contenido de $\mathrm{MO}$ de 1,5\%, esta diferencia puede atribuirse a que los productores incluyen en su manejo de suelos, la incorporación de los residuos de cosechas de las plantaciones que cumplen su ciclo productivo. Este parámetro presentó diferencias estadísticamente significativas dentro del rango 2,4 a 2,9\%, entre las localidades no se presentaron diferencias significativas. La localidad que mostró el mayor contenido de $\mathrm{MO}$ fue Villanueva con 3,39\% y la localidad de Reposito presentó el menor valor con 2,1\%. Las localidades con contenidos promedios fueron Tinajones y Reposo con 2,8\%. La distribución espacial de la materia orgánica muestra que el $87 \%$ (7.570 ha) presentan contenido entre $2,0-3,0 \%$, el $24,1 \%$ del área (15.982) presenta contenidos entre 2,5 $3,0 \%$; indicando que loscontenidos de materia orgánica en esta zona son buenos (Figura 3b). Los contenidos altos de MO en la parte norte, se debe probablemente al efecto de factores climáticos como temperatura, brillo solar y óptimos niveles humedad regulados por la lluvias, que hacen que la descomposición (humificación y mineralización) ocurra a tasas mayores comparada con otras zonas del área.

Según Stevenson (1984), la mineralización, de la materia orgánica, se inicia a $10^{\circ} \mathrm{C}$, y aumenta hasta alcanzar su máximo entre 30 y $40{ }^{\circ} \mathrm{C}$. De esto resulta que a temperaturas relativamente bajas se producen más residuos de los que se mineralizan y a temperaturas mayores de 25$28{ }^{\circ} \mathrm{C}$, la $\mathrm{MO}$ disminuye, lo que implica que la temperatura crítica de aproximadamente 25 ${ }^{\circ} \mathrm{C}$ es decisiva en la producción y degradación de los restos vegetales. Temperaturas bajo, el nivel crítico permiten una acumulación de materia orgánica con la mejora de una serie de propiedades de los suelos. Cuando las temperaturas son excesivamente altas como ocurre en muchas zonas tropicales, se presenta una aceleración de la degradación de los restos vegetales en el suelo que causa graves problemas en su fertilidad. 
León et al. - Zonificación edafoclimática en cultivo de papaya

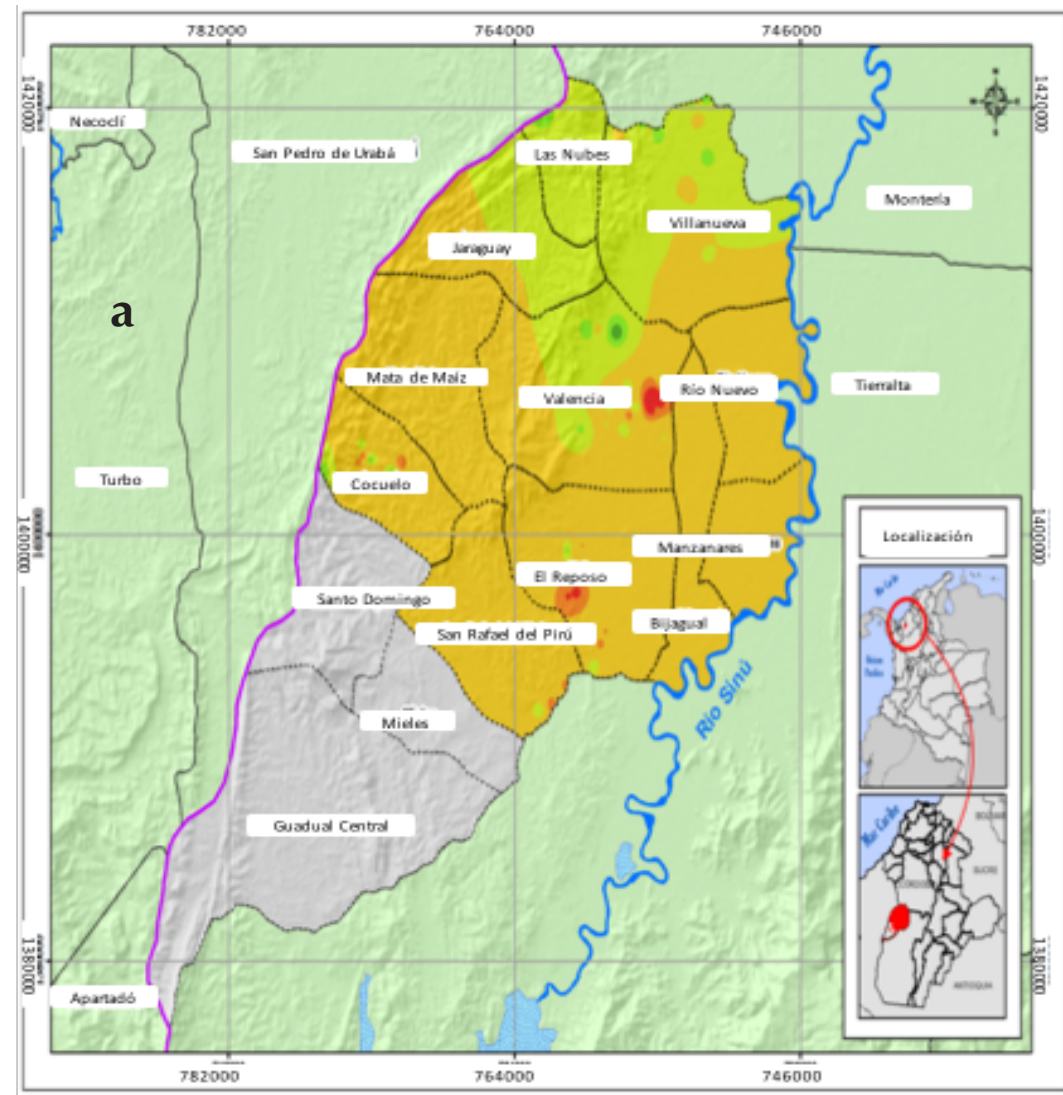

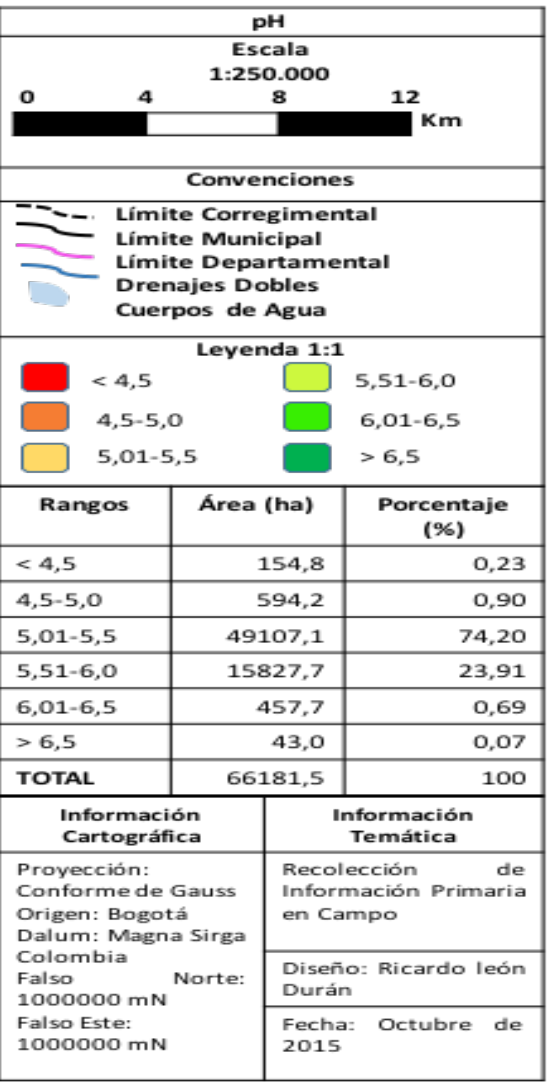

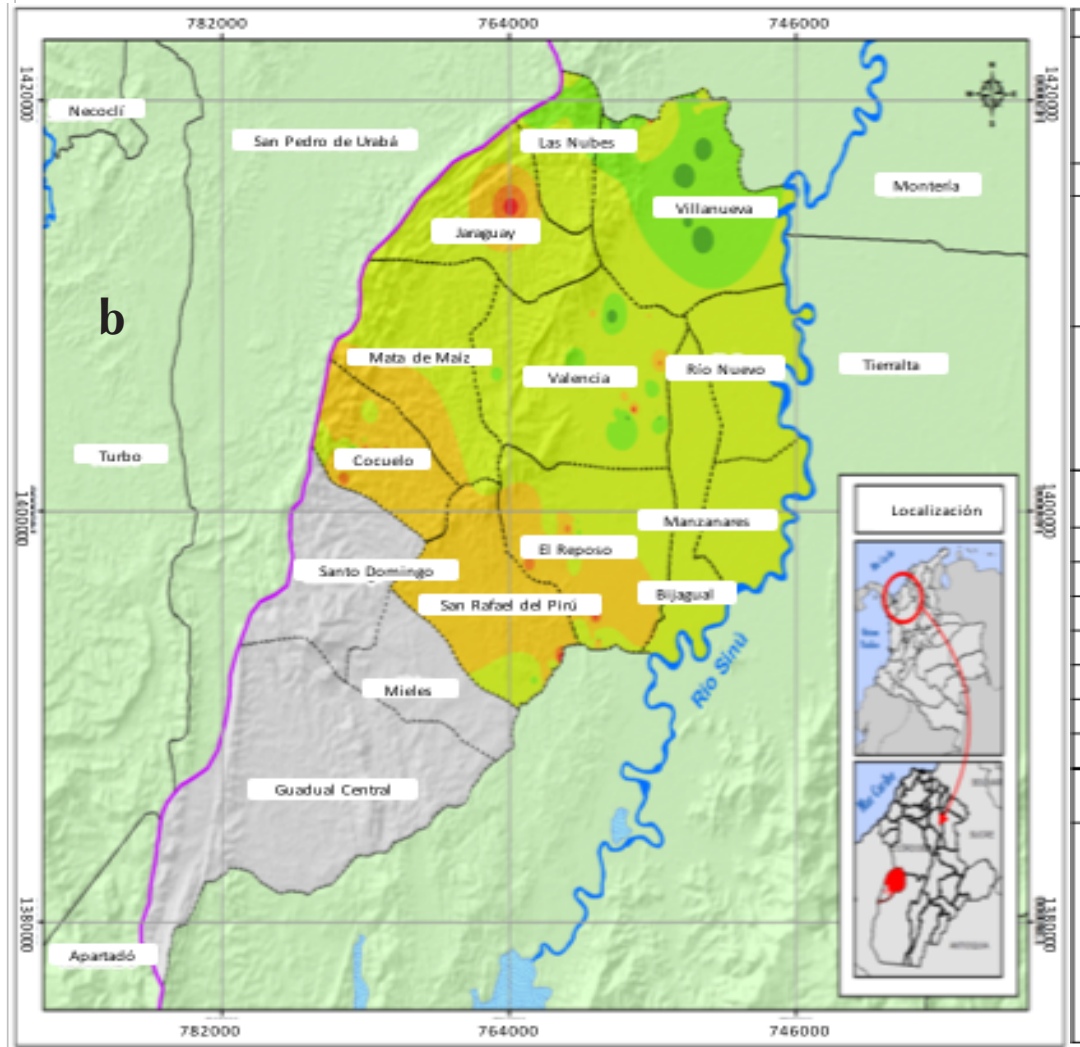

\begin{tabular}{|c|c|c|c|}
\hline \multicolumn{4}{|c|}{ Materia Orgánica (MO) } \\
\hline \multicolumn{4}{|c|}{$\begin{array}{c}\text { Escala } \\
\text { 1:250.ooo }\end{array}$} \\
\hline o & & & 12 \\
\hline \multicolumn{4}{|c|}{ Convenciones } \\
\hline \multicolumn{4}{|c|}{ 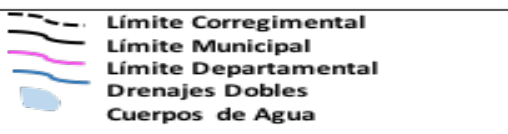 } \\
\hline \multicolumn{4}{|c|}{ Leyenda mg kg } \\
\hline \multicolumn{2}{|c|}{$<1,5$} & & $2,51-3,0$ \\
\hline & & $3,01-3,5$ \\
\hline \multicolumn{2}{|c|}{$2,01-2,5$} & & $>3,5$ \\
\hline Rangos & \multicolumn{2}{|c|}{ Área (ha) } & $\begin{array}{l}\text { Porcentaje } \\
(\%)\end{array}$ \\
\hline$<1,5$ & \multicolumn{2}{|c|}{74,6} & 0,11 \\
\hline $1,5-2,0$ & \multicolumn{2}{|c|}{496,4} & 0,75 \\
\hline $2,01-2,5$ & \multicolumn{2}{|c|}{15982,0} & 24,15 \\
\hline $2,51-3,0$ & \multicolumn{2}{|c|}{41568,0} & 62,84 \\
\hline $3,01-3,5$ & \multicolumn{2}{|c|}{7564,6} & 11,43 \\
\hline$>3,5$ & \multicolumn{2}{|c|}{476,3} & 0,72 \\
\hline TOTAL & \multicolumn{2}{|c|}{66181,5} & 100 \\
\hline \multicolumn{2}{|c|}{$\begin{array}{l}\text { Información } \\
\text { Cartográfica }\end{array}$} & \multicolumn{2}{|c|}{$\begin{array}{c}\text { Información } \\
\text { Temática }\end{array}$} \\
\hline \multirow{3}{*}{\multicolumn{2}{|c|}{$\begin{array}{l}\text { Proyección: } \\
\text { Conforme de Gauss } \\
\text { Origen: Bogotá } \\
\text { Dalum: Magna Sirga } \\
\text { Colombia } \\
\text { Falso } \\
1000000 \mathrm{mN} \\
\text { Falso Este: } \\
1000000 \mathrm{mN}\end{array}$}} & \multicolumn{2}{|c|}{$\begin{array}{l}\text { Recolección de } \\
\text { Información Primaria } \\
\text { en Campo }\end{array}$} \\
\hline & & \multicolumn{2}{|c|}{$\begin{array}{l}\text { Diseño: Ricardo león } \\
\text { Durán }\end{array}$} \\
\hline & & \multicolumn{2}{|c|}{$\begin{array}{l}\text { Fecha: Octubre de } \\
2015\end{array}$} \\
\hline
\end{tabular}

Figura 3. Nivel de $\mathrm{pH}(\mathrm{a})$, contenido de $\mathrm{MO}$ (b) y distribución espacial de $\mathrm{pH}$ y $\mathrm{MO}$ en diferentes localidades de Valencia, Córdoba, Colombia. 


\section{Zonificación}

\section{para el cultivo de papaya}

La zonificación edafoclimática para el cultivo de la papaya en la zona norte del municipio de Valencia es una herramienta de gestión para el desarrollo sostenible regional, en cuanto ofrece un marco de referencia a futuro para el ordenamiento territorial acerca de los factores biofísicos que caracterizan la capacidad de uso y aptitud productiva. Esta área comprende $66.181,5$ ha que se distribuyen en 10 corregimientos del municipio de Valencia. La distribución espacial en el área de referencia de las 4 categorías de aptitud; las zonas muy óptimas se caracterizan por presentar condiciones de climatológicas ideales para el desarrollo de las plantas en cuanto a pluviosidad, temperatura, humedad relativa y radiación solar, favoreciendo la floración, amarre y fructificación; igualmente las condiciones físicas, químicas y de acidéz de los suelos en esta zona favorecen el crecimiento y desarrollo de raices, como también, la disponibilidad y absorción de nuetrientes lo que puede generar mayor productividad $y$ sostenibilidad del cultivo (A1) indicadas por el SIG representan el 4,6\% equivalentes a aproximadamente a 3.050 ha, ubicadas en la parte norte del área, las zonas óptimas (A2) abarcan el 55\% del área equivalentes a 36.440,7 ha, ubicadas en el sector central del área de referencia, las zonas ligeramente óptimas (A3), cubren un área de $23.533,6$ ha y representan el $35,5 \%$ del área total, ubicadas en la parte suroriental y el $4,7 \%$ equivalente a $3.156,5$ ha fueron categorizadas como ligeramente óptimas (A4), están ubicadas en el sector suroccidental de la zona estudiada (Figura 4). De la Rosa (2008), afirma que la información de suelos es un elemento crucial para fundamentar decisiones agrícolas realmente sostenibles; siendo la evaluación agroecológica, la interface lógica entre dicha información básica y la toma de decisiones sobre el uso y manejo de la tierra.

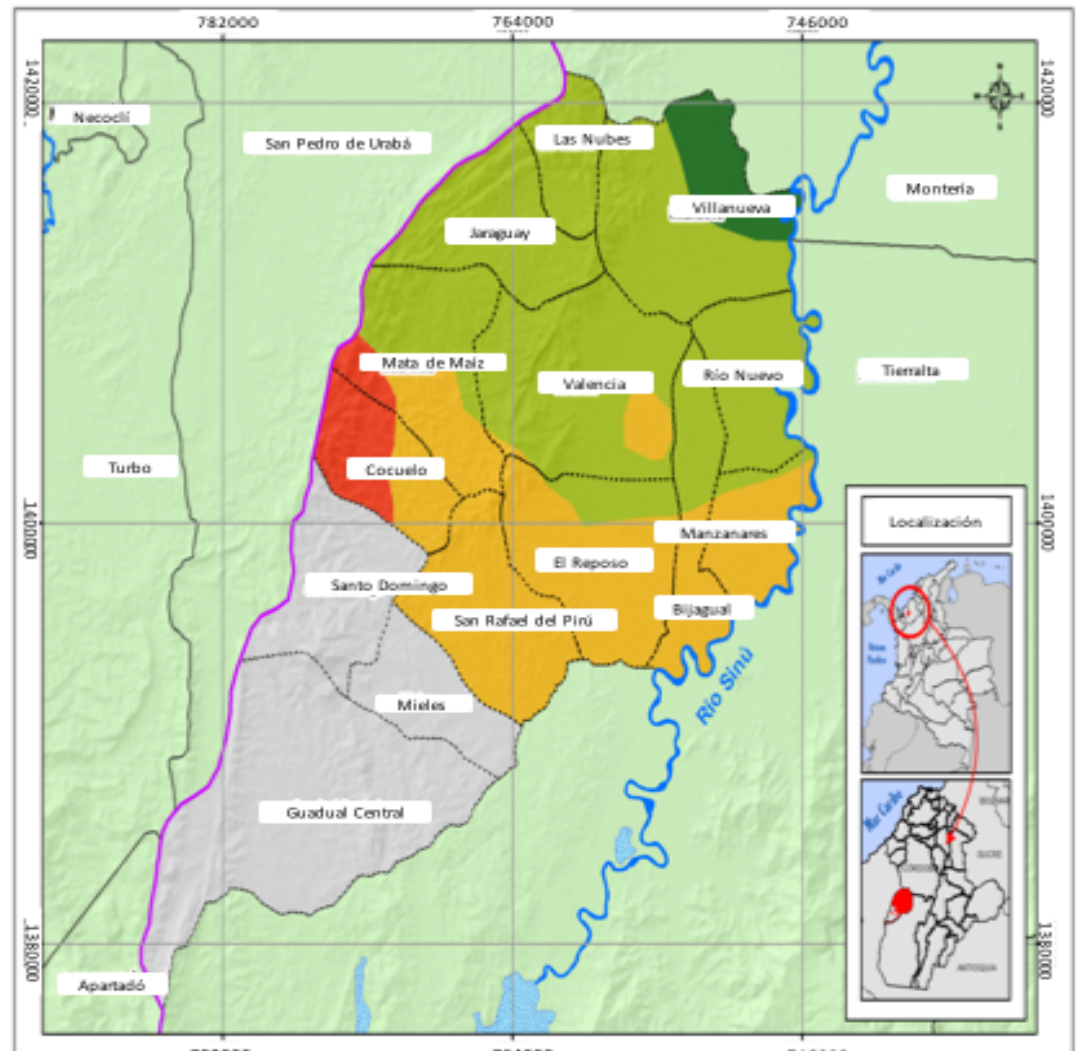

\begin{tabular}{|c|c|c|}
\hline \multicolumn{3}{|c|}{ Zonificación Edafoclimática para el Cultivo de Papaya } \\
\hline \multicolumn{3}{|c|}{ Escala } \\
\hline \multirow{2}{*}{\multicolumn{3}{|c|}{$\begin{array}{r}1: 250.000 \\
8\end{array}$}} \\
\hline & & \\
\hline \multicolumn{3}{|c|}{ Convenciones } \\
\hline \multirow{4}{*}{\multicolumn{3}{|c|}{$\begin{array}{l}\text { Limite Corregimental } \\
\text { Limite Municipal } \\
\text { Limite Departamental } \\
\text { Drenajes Dobles } \\
\text { Cuerpos de Agua }\end{array}$}} \\
\hline & & \\
\hline & & \\
\hline & & \\
\hline \multicolumn{3}{|c|}{ Leyenda } \\
\hline \multicolumn{3}{|l|}{ Muy Óptima } \\
\hline \multicolumn{3}{|l|}{ Óptima } \\
\hline \multicolumn{3}{|c|}{ Ligeramente Óptima } \\
\hline \multicolumn{3}{|c|}{ Moderadamente Óptima } \\
\hline Rangos & Área (ha) & Porcentaje (\%) \\
\hline Muy Óptima & 3050,6 & 4,61 \\
\hline Óptima & 36440,7 & 55,06 \\
\hline Ligeramente Óptima & 23533,6 & 35,56 \\
\hline Moderadamente Óptima & 3156,5 & 4,77 \\
\hline TOTAL & $66.181,5$ & 100 \\
\hline Información Cartográfica & \multicolumn{2}{|c|}{ Información Temática } \\
\hline \multirow{2}{*}{$\begin{array}{l}\text { Proyección: Conforme de } \\
\text { Gauss } \\
\text { Origen: Bogotá }\end{array}$} & \multicolumn{2}{|c|}{$\begin{array}{l}\text { Recolección de Información } \\
\text { Primaria en Campo }\end{array}$} \\
\hline & & \\
\hline \multirow{3}{*}{$\begin{array}{l}\text { Dalum: Magna Sirga } \\
\text { Colombia } \\
\text { Falso Norte: } 1000000 \mathrm{mN} \\
\text { Falso Este: } \\
1000000 \mathrm{mN}\end{array}$} & \multirow{2}{*}{\multicolumn{2}{|c|}{ Diseño: Ricardo león Durán }} \\
\hline & & \\
\hline & \multicolumn{2}{|c|}{ Fecha: Octubre de 2015} \\
\hline
\end{tabular}

Figura 4. Zonificación edafoclimática del cultivo de papaya en Valencia Córdoba 


\section{CONCLUSIONES}

De las 66181,5 ha del área de estudio, la zona categorizada como muy óptima presenta condiciones de climáticas y edáficas adecuadas para el desarrollo del cultivo de la papaya (A1), comprende 3.050 ha, al norte del área de estudio, las zonas óptimas (A2) abarcan 36.440,7 ha, ubicadas en el sector central, para as zonas ligeramente óptimas (A3), alcanzan 23.533,6 ha, ubicadas en la parte suroriental y las zonas ligeramente óptimas (A4) engloban 3.156,5 ha ubicadas en el sector suroccidental de la zona estudiada.

Las variables climáticas analizadas (precipitación, temperatura y humedad relativa) en elárea deestudió mostraron rangos adecuados para el desarrollo del cultivo de la papaya.

La evaluación de tierras por medio de la tecnología de los SIGs, demostró ser una herramienta de gran utilidad para la representación de la distribución espacial y la determinación de la aptitud productiva de suelos para el cultivo de papaya.

El 99,24\% del área total de estudio presentó niveles de pH menores a 6,0, caracterizándo la zona como prevalentemente ácida, el $12,15 \%$ del área de estudio mostró contenidos de $\mathrm{MO}$ superiores al 3\%, y el 43,94, registró contenidos de $\mathrm{Al}^{3+}$ superiores a $4 \mathrm{cmol}_{\mathrm{c}} \mathrm{kg}^{-1}$.

\section{REFERENCIAS}

Arango, L., Román, C., Salamanca, C., Almansa, E., Bernal, J., León, G., Rey, V., Ariza, M. y Gómez, P. 1999. El cultivo de papaya en los Ilanos orientales de Colombia. Corpoica - SENA Asohofrucol. Manual de asistencia técnica No 04. Villavicencio. 99p.

Barros, J. y Troncoso, A. 2010. Atlas climatológico del Ecuador (Bachelor's thesis, QUITO/EPN/2010)
Casierra, F,yAguilar, O.2007. Estrés poraluminio en las plantas: reacciones en el suelo, síntomas en vegetales y posibilidades de corrección. Revista Colombiana de Ciencias Hortícolas, 1(2), 246-256.

Campostrini, E. y Glenn, D. 2007. Ecophysiology of papaya: a review. Brazilian Journal of Plant Physiology, v.19, p.413-424, http://dx.doi.org/10.1590/ S $1677-04202007000400010$.

Combatt-Caballero, E. Novoa-Yánez, R. y Barrera-violeth, J. 2012. Caracterización química de macroelementos en suelos cultivados con plátano (Musa AAB Simmonds) en el departamento de Córdoba, Colombia. Acta Agronómica, 61(2), 166-176.

Clemente, H. y Marler, T. 2001. Trade winds reduce growth and influence gas exchange patterns in papaya seedlings. Annals of Botany, v.88, p.379-385, http:// dx.doi.org/10.1006/anbo.2001.1479.

Cristancho, J., Hana, M., Syed, R. y Rafii, M. 2010. Variations in oil palm (Elaeis guineensis Jacq.) progeny response to high aluminum concentrations in solution culture. Plant Biology, 13(2): 33-42.

Chuvieco, S. 2002. Teledetección ambiental. La observación de la tierra desde el espacio, Ed. Ariel, Barcelona España, 2002.

De la Rosa, D. 2008. Evaluación agroecológica de suelos: para un desarrollo rural sostenible (Contiene CD-Rom). Mundi-Prensa Libros.

Espinosa, J., y Roquera, A. 2007. Zonificación agroecológicadel cultivodepapa(Solanum tuberosum) en las provincias de: Bolívar, Cotopaxi, Chimborazo y Tungurahua. Rev. Rumipamba, 21(1), 54-56.

FAO. Organización de las Naciones Unidas para la Infancia y la Agricultura. 1997. Zonificacion Agro-ecologica, Food and Agriculture Organizationof the united Nations, Servicio de Recurso, Manejo y Conservacion de Suelos Boletín de Suelos número 73, Direccion de Fomento de Tierras y Aguas. 
Garea, E., Soto, F. y Vantour, A. 2008. "Zonificación en condiciones de agroecológica mediante métodos de análisis espacial", Revista internacional de ciencias de la tierra, (127), 36-40.

Gómez, Z. 2013. Diseño de un modelo funcional de distribución y operaciones logísticas para empresas productoras y comercializadoras de papaya de los municipios de Roldanillo y La Unión en el departamento del Valle del Cauca. Tesis de Maestría, Universidad Autónoma de Occidente. Cali. Colombia

Hue, N., Craddock, G. y Adams, F. 1986. Effect of Organic Acids on Aluminum Toxicity in Subsoils 1. Soil Science Society of America Journal, 50(1), 28-34.

Hueso, J., Salinas, I. y Cuevas, J. 2015. Cajamar ADN Agro. El cultivo de la papaya. Fichas de transferencia, 10p.

Instituto Colombiano Agropecuario ICA, 2011. Resolución 04913. Por medio de la cual se declara un área localizada en los municipios de Valencia y Tierralta en la zona del Alto Sinú del departamento de Córdoba, como área de Baja Prevalencia para Anastrepha sp. complejo fraterculus W. y Ceratitis capitata W.

Instituto Geográfico Agustín Codazzi IGAC. 2009. Estudio general de suelos y zonificación de tierras del departamento de Córdoba. 501 p.

Jeyakumar, P., Kavino, M. y Kumar, N. 2007. Physiological performance of papaya cultivars under abiotic stress conditions. Acta Horticulturae, v.740, p.209-216.

Jiménez, A., Vargas, V., Salinas, W., Aguirre, M. y Rodríguez, D. 2004. Aptitud agroecológica para el cultivo de la caña de azúcar en el sur de Tamaulipas, México. Investigaciones geográficas, (53), 58-74.
Kerven, G, Edwards, D, Asher, C, Hallman, P, y Kokot,S.1989. Determinación dealuminio en solución de suelo. 2. Procedimientos colorimétricos a corto plazo para la medición de aluminio monomérico inorgánico en presencia de ligandos de ácido orgánico. Soil Research, 27 (1), 91 102. https://doi.org/10.1071/SR9890091

León, J. 2013. Cultivos potenciales de interés para las Américas. Revista de Geografía Agrícola, 50-51p.

Moreno, A. 2005. Sistemas y análisis de la información geográfica, Madrid, Editorial RA-MA, 895 p

Palencia, G., Mercado, T. y Combatt, E. 2006. Estudio Agroclimático del Departamento de Córdoba (No. P01-295). Universidad de Córdoba (Colombia), Facultad Ciencias Agrarias.

Rivera, Y., Moreno, L., Herrera, M. y Romero, H. 2016. La toxicidad por aluminio $(\mathrm{Al} 3+)$ como limitante del crecimiento y la productividad agrícola: el caso de la palma de aceite. Palmas, 37(1), 11-23),

Suárez, G., Bacallao, R., Soto, F. y Caballero, N. 2013. Bases para la zonificación agroecológica en el cultivo del cacao (Theobroma cacao, Lin) por medio del criterio de expertos. Cultivos Tropicales, 34(2), 30-37.

Vázquez, M. 2006. Calcio y magnesio. Acidez y alcalinidad de los suelos. En Fertilidad de suelos y fertilización de cultivos. H.E. Echeverría y F.O. García (eds). Editorial INTA. Buenos Aires. Argentina. pp. 161-189.

Venero, S., y Gicli, M. 2014. Apuntes sobre la zonificación agroecológica de los cultivos. Particularidades en Cuba. Cultivos Tropicales, 35(4), 36-44.

Stevenson, F. 1984. Humus Chemistry. Genesis, Composition, Reactions. John Wiley and Sons. New York. 443 p. 